\title{
Robustness of high-fidelity Rydberg gates with single-site addressability
}

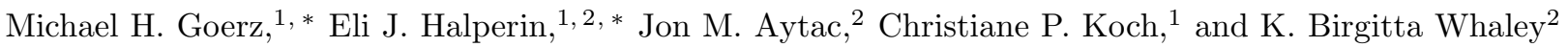 \\ ${ }^{1}$ Theoretische Physik, Universität Kassel, Heinrich-Plett-Str. 40, D-34132 Kassel, Germany \\ ${ }^{2}$ Department of Chemistry, University of California, Berkeley, California 94720, USA
}

(Dated: May 31, 2022)

\begin{abstract}
Controlled phase (CPHASE) gates can in principle be realized with trapped neutral atoms by making use of the Rydberg blockade. Achieving the ultra-high fidelities required for quantum computation with such Rydberg gates is however compromised by experimental inaccuracies in pulse amplitudes and timings, as well as by stray fields that cause fluctuations of the Rydberg levels. We report here a comparative study of analytic and numerical pulse sequences for the Rydberg CPHASE gate that specifically examines the robustness of the gate fidelity with respect to such experimental perturbations. Analytical pulse sequences of both simultaneous and stimulated Raman adiabatic passage (STIRAP) are found to be at best moderately robust under these perturbations. In contrast, optimal control theory is seen to allow generation of numerical pulses that are inherently robust within a predefined tolerance window. The resulting numerical pulse shapes display simple modulation patterns and their spectra contain only one additional frequency beyond the basic resonant Rydberg gate frequencies. Pulses of such low complexity should be experimentally feasible, allowing gate fidelities of order 99.90 - 99.99\% to be achievable under realistic experimental conditions.
\end{abstract}

PACS numbers: 02.30.Yy,03.67.Bg,37.10.Jk

\section{INTRODUCTION}

Rydberg states of trapped neutral atoms provide an attractive platform for realizing quantum information processing, offering a strong interaction between relatively distant and otherwise non-interacting atoms that may be switched on and off with focused lasers [1]. Proposals have been made for quantum gates with both addressable and non-individually addressable single atom qubits 2] as well as with atomic ensembles [3, 4]. These schemes typically employ resonant excitation and make use of the Rydberg blockade to generate controlled phase relationships between logical qubit states that are typically defined as hyperfine states of the ground electronic atomic manifold. Progress in trapping and manipulating single atoms in dipole traps and optical tweezers has enabled experimental validation of the key theoretical concepts of the Rydberg blockade [5, 6], as well as subsequent use of this to generate entanglement between trapped atoms in these configurations [7, 8]. The latter study also demonstrated a low fidelity version of a controlled not (CNOT) gate based on the Rydberg blockade. Parallel to this, several groups have developed the capability to form arrays of trapped atoms in optical lattices that are characterized by single site occupancy and addressability [9-14], thereby opening the path to large scale quantum information processing with atomic qubits.

Despite these conceptual and experimental advances, realization of high fidelity quantum logic gates between such trapped neural atoms has remained elusive, due to the significant challenges involved in coherently control-

\footnotetext{
${ }^{*}$ These authors contributed equally.
}

ling and manipulating optically trapped atoms. Twoqubit gates relying on controlled use of dipolar interactions between atoms in Rydberg states have the potential of being fast, but are subject to a number of intrinsic and technical sources of error that can restrict both the achieved fidelity and speed of operation. An important source of intrinsic error specific to Rydberg gates is the lifetime of the atoms in the Rydberg states while technical errors may derive from a number of experimental factors, as discussed recently in [15]. For the nonindividually addressable implementation of the Rydberg gate protocol in [2], atomic motion can also play a significant role in limiting the fidelity [16, 17]. The role of these and other factors limiting gate fidelities have been studied theoretically for Rydberg gate schemes involving both analytic pulse sequences [15, 18] and, for the non-addressable protocol of [2], numerically optimized pulses [16, 17]. These studies indicate that gates with errors of the order of $10^{-3}$ might be achieved with suitable choice of atoms and qubit levels. However, no study of the robustness of two-bit gates with respect to errors has been made, although such robustness with regard to fluctuations of both intrinsic and technical parameters is a critical desiderata of experimental studies. In this work we remedy this with a systematic study of the robustness of both analytic and numerical pulse sequences with respect to the primary technical fluctuating parameters, namely pulse timing, pulse amplitude, and two-photon detuning.

Another desiderata for quantum information processing is the realization of fast gates. While proposals have been made to mitigate the effects of intrinsic errors in Rydberg gates using adiabatic passage techniques [19], the resulting pulse sequences typically result in relatively long gate times of $\mu \mathrm{s}$ or longer [4, 20], which is disadvan- 
tageous for quantum computation schemes that generally require large numbers of gates. Prospects for achieving gates on ns timescales have been reviewed in 21] and for the non-addressable protocol of [2], optimal control theory has been used to characterize bounds on the shortest possible gate time [22], corresponding to a "quantum speed limit" for performing the gate [23].

The remainder of the paper is constructed as follows. Section [II summarizes the atomic level structure and qubit model, as well as basic components of the CPHASE gate implementation with Rydberg states of individually addressable atoms. For technical reasons, the transition to the Rydberg level is via an intermediary state. In Section III we first analyze the performance of three forms of analytic pulse sequences. The first is the original $\pi-2 \pi-\pi$ sequence of [2], where each pulse consists of a simultaneous pulse pair realizing a two-photon transition to a Rydberg state. The second is a fully adiabatic version of this, in which each simultaneous pulse pair is replaced by a STIRAP pulse pair, and the third is a mixed scheme in which only the $\pi$ pulses are replaced by STIRAP pulse pairs. These different schemes are then compared in their robustness with respect to intrinsic experimental parameters. We find that the mixed scheme is the most robust of these analytic approaches, due to its selective use of STIRAP on the control qubit only. However, all STIRAP based schemes require either large pulse amplitudes or exceedingly long pulse times. Section IV demonstrates the benefits offered by numerical optimal control calculations in generating pulse sequences. We first determine the optimal pulses for a given pulse duration using the Krotov method [24 26] within a density matrix formulation for the open quantum system dynamics, taking spontaneous emission into account. Optimization for pulses robust to fluctuations in pulse amplitude and Rydberg energies (due, e.g., to stray fields) is then made over an ensemble of Hamiltonians within an experimentally relevant tolerance window. We find that optimal control yields systematically higher gate fidelities than all analytic approaches, showing improvement of an order of magnitude to reach gate errors of order $10^{-4}$ for equivalent gate times. Most importantly, optimal control can deliver gate performance that is also extremely robust with respect to experimental fluctuations, with the gate error staying below or at the order of $10^{-3}$ even for large fluctuations. Using optimal control we can also significantly shorten the total gate duration, to $\sim 100 \mathrm{~ns}$, approaching the quantum speed limit for these systems, without loss in either robustness or fidelity. The resulting numerical pulse spectra are surprisingly simple and allow the error threshold for fault tolerant computation to be reached at the price of a small increase in pulse complexity relative to the analytic sequences.

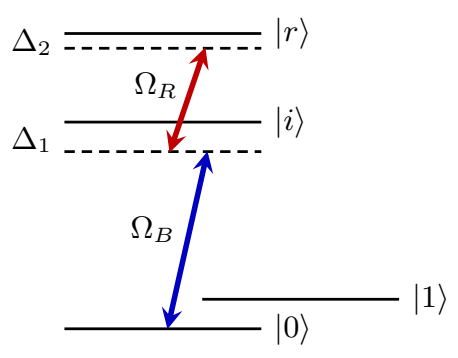

FIG. 1: (Color online) Level scheme for a single atom. The color scheme given here, blue for the lower transition and red for the upper one, is used throughout the figures of this paper.

$$
\begin{array}{ll}
\text { Single-Photon Detuning } & \Delta_{1}=1.273 \mathrm{GHz} \\
\text { Two-Photon Detuning } & \Delta_{2}=00 \mathrm{MHz} \\
\text { Qubit Energy } & E_{1}=9.100 \mathrm{GHz} \\
\text { Interaction Energy } & u=57.26 \mathrm{MHz}
\end{array}
$$

TABLE I: System Parameters

\section{MODEL}

We consider two cesium atoms trapped in an optical lattice with single-site addressability. The qubit states are encoded in hyperfine levels of the ground state, $|0\rangle=\left|6{ }^{2} S_{1 / 2}, F=3\right\rangle,|1\rangle=\left|6{ }^{2} S_{1 / 2}, F=4\right\rangle$. For practical reasons, the Rydberg level, here $|r\rangle=\left|50 D_{3 / 2}\right\rangle$, is accessed by a two-photon transition via an intermediate state, $|i\rangle=\left|7 P_{3 / 2}\right\rangle$. In the basis $\{|0\rangle,|1\rangle,|i\rangle,|r\rangle\}$, the Hamiltonian for a single atom, using a two-photon rotating-wave approximation [27], reads

$$
\hat{\mathbf{H}}_{1 \mathrm{q}}=\left(\begin{array}{cccc}
0 & 0 & \Omega_{B}(t) & 0 \\
0 & E_{1} & 0 & 0 \\
\Omega_{B}(t) & 0 & \Delta_{1} & \Omega_{R}(t) \\
0 & 0 & \Omega_{R}(t) & \Delta_{2}
\end{array}\right),
$$

where $\Omega_{B}(t), \Omega_{R}(t)$ are the Rabi frequencies of the 'blue' and 'red' pulses, cf. Fig. 1. and $\Delta_{1}, \Delta_{2}$ are the one-photon and two-photon detunings. The two atoms are kept at a distance of $5 \mu \mathrm{m}$ such that their interaction is negligible except when both atoms are in the Rydberg state. The Hamiltonian for the two atoms, including their Rydberg interaction, is written as

$$
\hat{\mathbf{H}}_{2 q}=\hat{\mathbf{H}}_{1 q} \otimes \mathbb{1}+\mathbb{1} \otimes \hat{\mathbf{H}}_{1 q}-u|r r\rangle\langle r r|,
$$

with interaction energy $u$. The parameters are summarized in Table I. Rabi frequencies of $\Omega_{B}=171.5 \mathrm{MHz}$ and $\Omega_{R}=148.4 \mathrm{MHz}$ have been implemented for this system and values up to $\sim 250 \mathrm{MHz}$ are expected to be experimentally feasible [28].

Resonant excitation of both atoms to the Rydberg state leads to an acceleration of the atoms due to the dependence of the Rydberg interaction strength on interatomic separation [2]. The minimum gate duration is then determined either by the inverse of the interaction, 
$u$, or by the period of the atomic motion in the trap [16]. The gate duration may be limited further by the inverse of the experimentally realizable Rabi frequencies.

We consider the Rydberg blockade regime which avoids resonant excitation into $|r r\rangle$. It corresponds to

$$
u \gg \Omega_{j} \quad(j=B, R)
$$

The original proposal of the Rydberg gate 2] in this regime requires the atoms to be individually addressable, and employs a sequence of three pulses: a $\pi$-pulse on the left atom, resulting in complete population transfer from $|0\rangle$ to $|r\rangle$, followed by a $2 \pi$-pulse on the right atom and another $\pi$-pulse on the left atom. If the qubits are initially in $|00\rangle$, a non-local phase is accumulated during the middle pulse because of the detuning of level $|r r\rangle$ due to the interaction, $u$, and we thus can execute a CPHASE gate.

\section{ANALYTIC PULSE SEQUENCES}

When a resonant two-photon transition is employed via an intermediate level, the two-level system $\{|0\rangle,|r\rangle\}$ for one atom in the original proposal [2] is replaced by $\{|0\rangle,|i\rangle,|r\rangle\}$. The $\pi$ and $2 \pi$ population flips can then be realized either with two simultaneous pulses: where $\Omega_{B}$ connecting $|0\rangle$ and $|i\rangle$ and $\Omega_{R}$ connecting $|i\rangle$ and $|r\rangle$ are driven contemporaneously; or via a STIRAP process: where $\Omega_{R}$ acts as a "Stokes" pulse, preceding but overlapping $\Omega_{B}$, the "pump" pulse. Both methods may be combined in a mixed scheme, where a STIRAP sequence is used for the $\pi$ flip acting on the left atom, while the $2 \pi$ flip on the right atom is realized using simultaneous pulses. The following sections discuss the merits and drawbacks of all three approaches, and numerically analyze the robustness with respect to pulse timing, fluctuations of the Rydberg level, and fluctuations of the pulse amplitude.

\section{A. Sequence of three simultaneous pulse pairs}

We first consider the realization of all population transfers using simultaneous pulse pairs. The pulses are of Blackman shape,

$$
S(t)=\frac{E_{0}}{2}(1-a-\cos (2 \pi t / T)+a \cos (4 \pi t / T)),
$$

with $a=0.16$ and $E_{0}$ the peak amplitude. This pulse shape is essentially identical to a Gaussian centered at $T / 2$ with a width of $\sigma=T / 6$, but, unlike the Gaussian, is exactly zero at $t=0$ and $t=T$. Other pulse shapes are possible.

A pulse sequence that realizes the two $\pi$-flips on the left atom and one $2 \pi$-flip on the right atom is shown in Fig. 2. Due to the large single photon detuning of $1.3 \mathrm{GHz}$, the

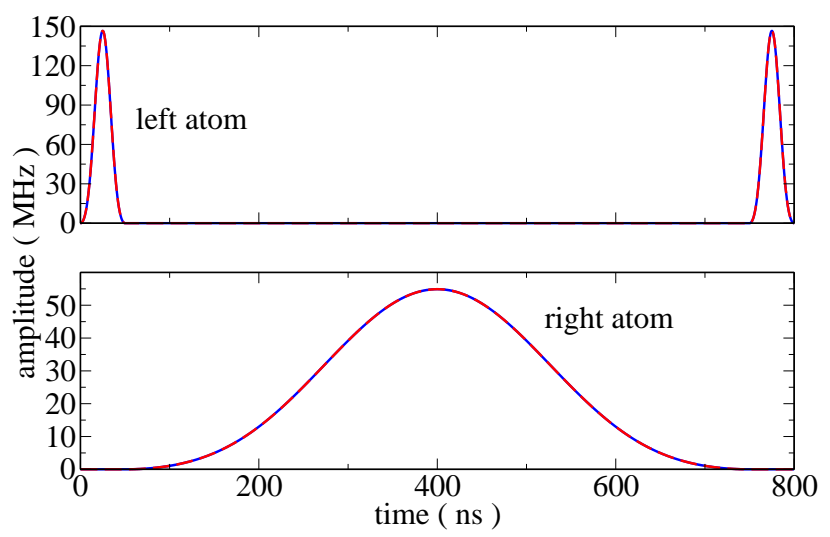

FIG. 2: (Color online) Three sequential Blackman pulse pairs implementing a CPHASE gate.

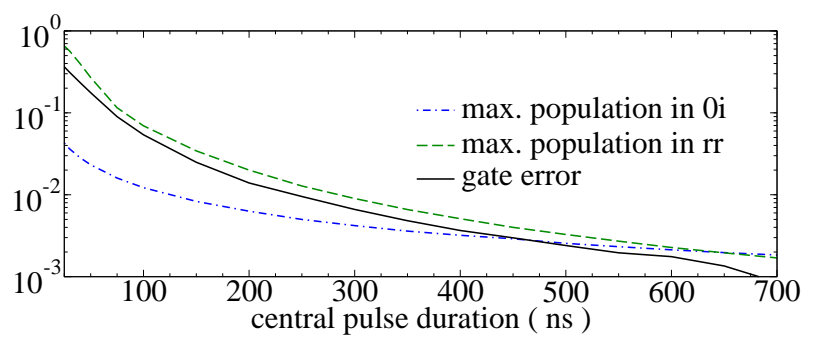

FIG. 3: (Color online) Quantum speed limit for the Rydberg gate using simultaneous Blackman pulse pairs. The time window is only that of the center $2 \pi$ pulse in the scheme. As a measure of the breakdown of the Rydberg blockade, the maximum population in the $|r r\rangle$ state during that pulse is shown, as well as the maximum population in the $|01\rangle$ state, as a measure of the breakdown of the adiabatic elimination of the intermediate level. Finally, we show the total gate error obtained when combining the center $2 \pi$ pulse of the given duration with two 50 ns $\pi$ pulses on the left atom.

intermediate level can be adiabatically eliminated. This places a restriction on the pulse amplitude,

$$
\Omega_{j} \ll \Delta_{1} \quad(j=B, R) .
$$

The $2 \pi$ pulse is more stringently restricted by the blockade condition in Eq. (3). With the pulse duration being inversely proportional to the pulse amplitude, both effects result in a quantum speed limit.

Quantitatively, the limitations are illustrated in Fig. 3 which shows the gate error (black solid line) vs. duration of the middle $2 \pi$ pulse, using a duration of $50 \mathrm{~ns}$ for the initial and final $\pi$ pulse. The gate error is defined as $1-F$ where $F$ is the gate fidelity,

$$
F=\frac{1}{20}\left(\left|\operatorname{tr}\left[\hat{\mathbf{O}}^{\dagger} \hat{\mathbf{U}}\right]\right|^{2}+\operatorname{tr}\left[\hat{\mathbf{U}}^{\dagger}\right]\right)
$$

with Ô the target CPHASE gate, and $\mathbf{U}$ the projection of the time evolution operator onto the logical subspace (Û 
is unitary if and only if there is no loss from that subspace at final time $T$ ). The breakdown of adiabatic elimination becomes apparent in the peak population of the $|0 i\rangle$ state (green dashed line), whereas a breaking of the Rydberg blockade is observed in the peak population in the $|r r\rangle$ state (blue dot-dashed line). Gate errors below $10^{-3}$ are only reached for pulse durations of $\geq 800 \mathrm{~ns}$. The gate time is dominated by the central $2 \pi$ pulse, which must be sufficiently weak to not break the Rydberg blockade. Already, the pulse amplitude is remarkably close to the interaction energy, pushing the limits of condition (3). Note that the choice of identical peak Rabi frequencies for the red and blue laser, $\Omega_{B \text {, max }}=\Omega_{R \text {, max }}$, is the only ratio possible to guarantee complete population inversion in a three-level system using simultaneous pulses when the intermediate level is adiabatically eliminated [27].

Population and phase dynamics obtained with simultaneous red and blue pulses is shown in figure 4. As described in section II the population undergoes a $\pi$ Rabi cycle on the left atom, followed by a $2 \pi$ pulse on the right atom, followed by a $\pi$ pulse on the left atom, cf. Fig. 4 $(\mathrm{a}, \mathrm{b}, \mathrm{c})$. The intermediate level receives almost no population and thus, for this time scale, spontaneous decay is not an issue. As can be seen from Fig. 4 (f), the non-local phase is accumulated in the $|00\rangle$ state entirely during the central $2 \pi$ pulse. Although the Rydberg blockade is not broken, and the population remains in $|r 0\rangle$, the state accumulates an additional phase due to the detuned pulse driving the transition out of $|r 0\rangle$. This additional phase is critical for the success of the gate.

\section{B. Sequence of STIRAP pulse pairs}

STIRAP is a popular scheme to achieve population transfer in three-level systems, avoiding population in the intermediate level at all times [29]. It is based on adiabatically following a dynamic dark state that does not contain an $|i\rangle$-component. In our setup, the scheme for transferring population from $|0\rangle$ to $|r\rangle$ is realized by first switching on the red laser, acting as a "Stokes" pulse, followed by the blue laser, acting as the "pump" pulse. The two pulses must overlap, but the process is robust with respect to the laser amplitude and the exact overlap of the pulses, as long as the condition for adiabatic following, roughly given by [29]

$$
\Omega_{j} \Delta \tau \gg 10 \quad(j=B, R)
$$

is met, where $\Delta \tau$ is the time for which the pulses overlap. Thus, for short pulses, large amplitudes are required. However, for a Rydberg gate, the blockade condition, Eq. (3), also needs to be fulfilled, limiting the maximum Rabi frequency. Therefore STIRAP can only employ comparatively long pulses for the center $2 \pi$ Rabi flip on the right atom.

In order to quantify violation of the blockade condition, we define the 'blockade efficiency', $B$, to be

$$
B=\max \left(P_{1 r}\right)-\frac{1}{2} P_{1 r}(T)-\left(\max \left(P_{r r}\right)-\frac{1}{2} P_{r r}(T)\right)
$$

where $T$ is the total time of the pulse sequence and $P_{1 r}$ and $P_{r r}$ are the population in $|1 r\rangle$ and $|r r\rangle$, respectively. $B$ takes values between zero and one, with one corresponding to a perfect blockade. Both maximum and final-time populations appear in $B$ because, in order to have full Rabi cycling, the Rydberg level must be fully populated (giving a maximum population of one) and then fully depopulated (giving a final population of zero), i.e., considering only the maximum population does not allow for distinguishing between $\pi$ and $2 \pi$ pulses. We only obtain $B=1$ when the population completes a $2 \pi$ cycle through $|1 r\rangle$ whenever the system begins in $|10\rangle$ but never reaches $|r r\rangle$ whenever the system begins in $|00\rangle$. The blockade condition, Eq. (3), depends on the peak amplitude of the pulses whereas the adiabaticity condition, Eq. (7), depends on the pulses' complete Rabi angle. For short pulses the Rabi angle will not be sufficiently large to satisfy the adiabaticity condition without requiring a peak amplitude so high that it will break the blockade. This is illustrated in Fig. 5 (top), where for small amplitudes both the maximum and final $|1 r\rangle$ populations rise together: the Rabi angle is less than $\pi$ (dashed green and dotted red lines). Then, as the final $|1 r\rangle$ population begins to fall such that the adiabaticity condition of STIRAP is better fulfilled, the blockade is broken, causing the drop in the blockade efficiency, cf. solid black line, concurrent with a rise in both the maximum and final $|r r\rangle$ populations (long-dashed blue and dot-dashed orange lines). In Fig. 5 (bottom), the maximum and final $|1 r\rangle$ populations rise together (green dashed and dotted red lines), but $|1 r\rangle$ is now fully depopulated, thus achieving full Rabi cycling, before breaking the blockade. This corresponds to the area where $B \approx 1$ seen in the graph. We do not see a rise in the maximum and final $|r r\rangle$ population until high peak amplitudes (long dashed blue and dot-dashed orange lines).

A corresponding sequence of STIRAP pulse pairs, using short pulses on the left atom and long pulses on the right atom, is shown in Fig. 6. In principle, the pulses on the left atom can be made arbitrarily short, at the expense of extremely large field amplitudes. Taking into account realistic restrictions on the available laser power, the gate time will generally become prohibitively large.

Even in the regime where both the adiabaticity condition and the blockade condition are fulfilled, the fidelity oscillates rapidly as a function of the peak amplitude, as seen in Fig. 7. These oscillations are due to phase errors induced by small population of the intermediate state $|r i\rangle$ during the central pulses in the blockade regime. They may be compensated using the techniques proposed in Ref. [4], though this does not address the fundamental issue of large gate time. 

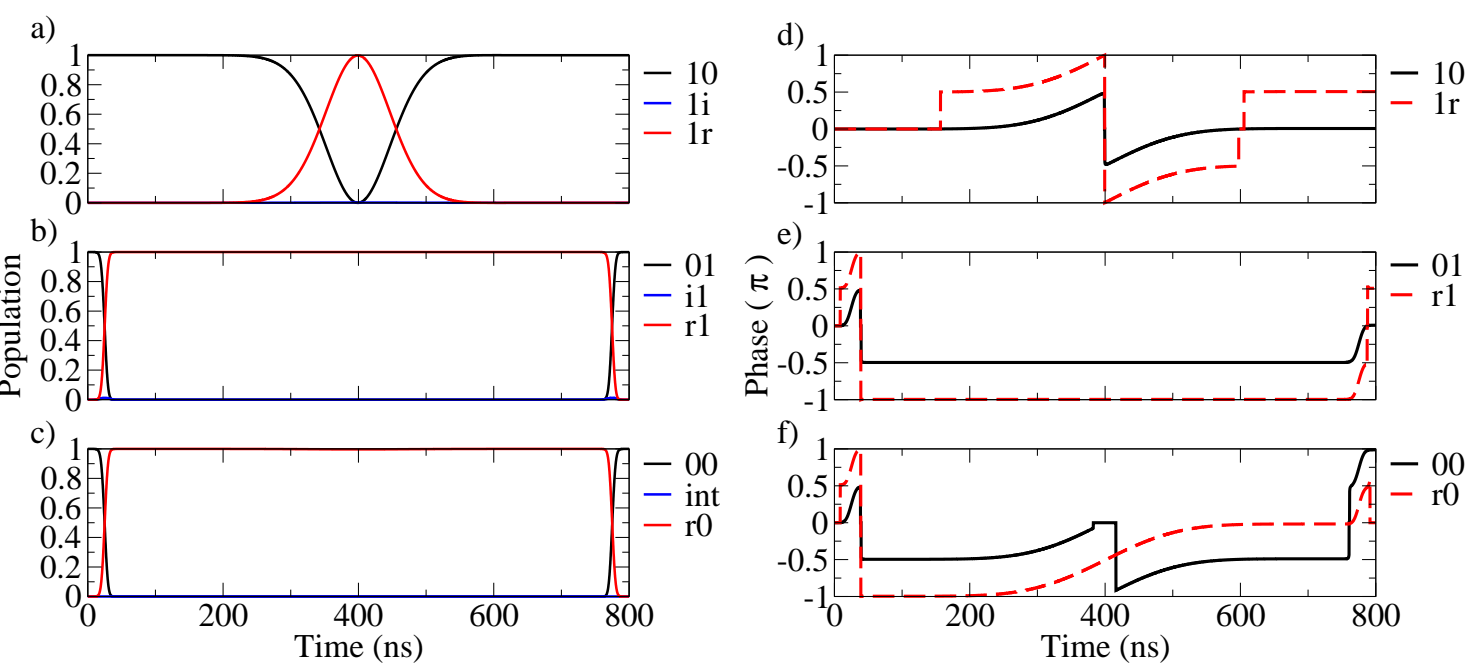

FIG. 4: Population and phase dynamics using the simultaneous pulses shown in Fig. 2

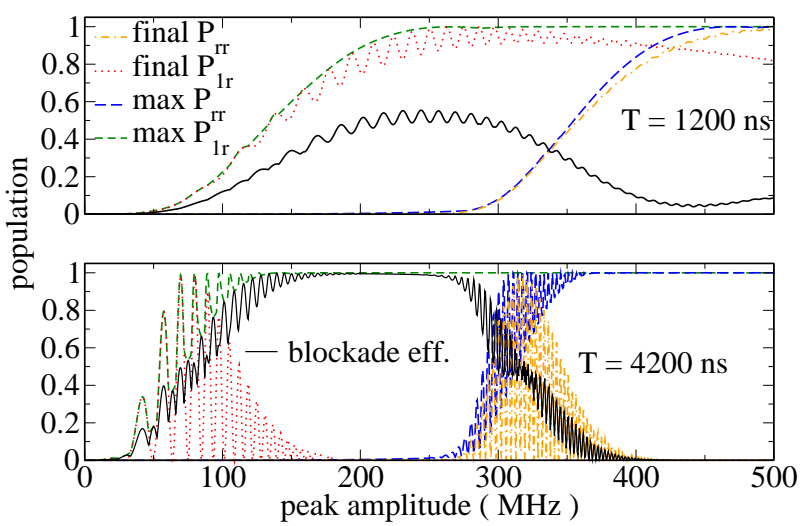

FIG. 5: (Color online) Breakdown of the Rydberg blockade for STIRAP: Only long gate durations allow for amplitudes that are sufficiently large to ensure adiabaticity in STIRAP while being small enough not to break the Rydberg blockade (lower panel).

\section{Mixed scheme: STIRAP- $\pi$-pulses and simultaneous $2 \pi$-pulses}

The primary drawbacks of the simultaneous pulses are the unwanted population in the intermediate level for the pulses acting on the left atom and a relatively large sensitivity of the pulses to variations in pulse area. On the other hand, the primary drawback of STIRAP is the breakdown of the Rydberg blockade, which results in employing an extremely long pulse acting on the right atom. This issue, however, is not present when using STIRAP for the pulses acting on the left atom. We therefore investigate a mixed scheme, consisting of STIRAP pulses to drive the $\pi$ rotations on the left atom and simulta-

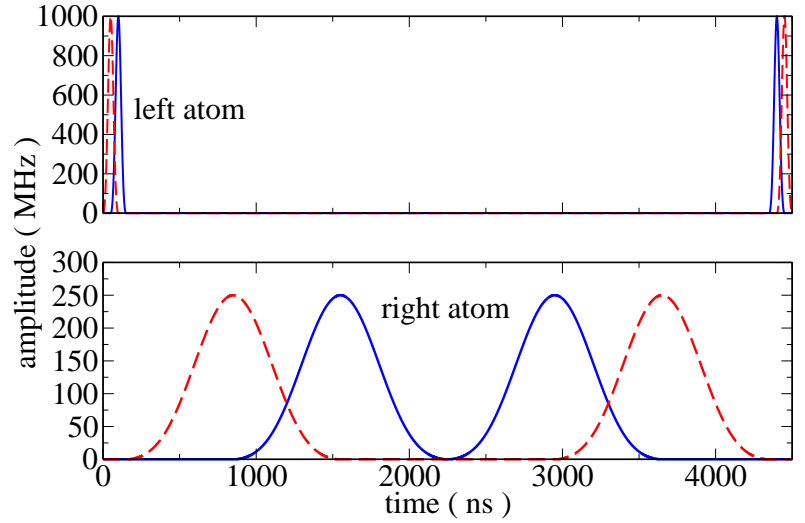

FIG. 6: A sequence of STIRAP pulse pairs to implement the Rydberg CPHASE gate: While the pulses acting on the left atom can be made very short (limited effectively by the power of the driving laser), the pulses acting on the right atom need to be sufficiently long to avoid breaking the Rydberg blockade.

neous pulses to drive the $2 \pi$ rotation on the right atom, cf. Fig. 8 By doing so we use each method where it is most effective. Furthermore, the pulses on the left and right atom can be overlapped without any appreciable loss in fidelity. This is because the pulses acting on the right atom only drive significant population transfer during the central third of the pulses. As long as the left atom is populated by the time the amplitude of the pulses acting on the right atom becomes significant, the blockade is still effective. The two STIRAP pulses acting on the left atom, that bookend the central pulses acting on the right atom, are moved in towards the center. In fact the pulses can be compressed quite far: By overlapping the STIRAP pulses with the central pulses for $250 \mathrm{~ns}$, cf. 


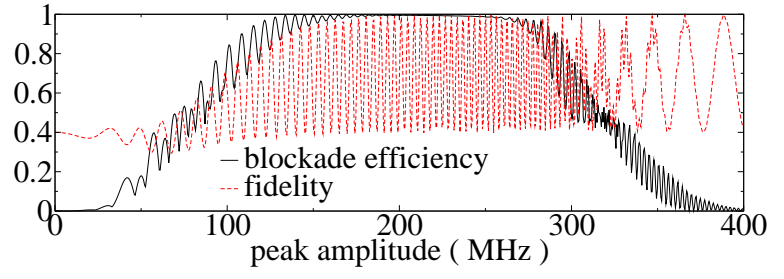

FIG. 7: (Color online) Gate fidelity for the Rydberg gate using STIRAP pulse pairs: even for amplitudes for which the blockade condition, Eq. (3), is fulfilled, the gate fidelity may be low due to improper phase alignment.

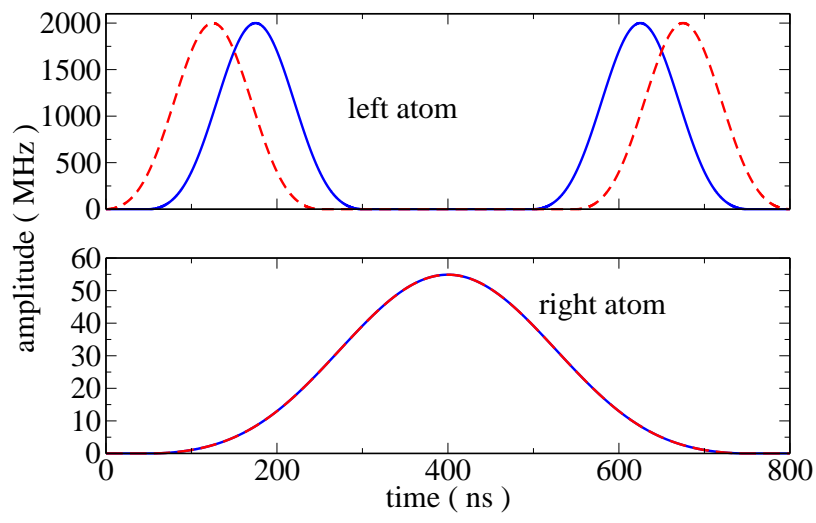

FIG. 8: (Color online) Mixed scheme: STIRAP pulse pairs for robust population transfer on the left atom, and simultaneous pulses for the $2 \pi$ rotation of the right atom.

Fig. 8, the gate duration can be reduced from $1300 \mathrm{~ns}$ to $800 \mathrm{~ns}$. The gate duration in the mixed scheme is limited by the laser power available for driving the left atom.

\section{Robustness}

For all three variants of pulse sequences, the gate fidelity in an actual experiment will be compromised by noise and experimental inaccuracies. We consider in the following three main sources of errors: inaccuracies in timing between the pulses acting on the left and right qubit, inaccuracies in pulse amplitudes, and fluctuations of the Rydberg level due to, e.g., the presence of DC electric fields [21]. The latter results in a non-zero twophoton detuning. To analyze the robustness with respect to all of these fluctuations, we determine the expectation value of the gate fidelity under the assumption that the timing offset, the transition dipole, and the two-photon detuning differ from the optimal values by $\Delta_{\text {time }}, \Delta_{\Omega}$, and $\Delta_{\text {ryd }}$ drawn from a Gaussian distribution centered at 0 of width $\sigma_{\text {time }}, \sigma_{\Omega}$, and $\sigma_{\text {ryd }}$, respectively. For the pulse amplitudes, the variation is given in percent of the original amplitudes. The expectation value of the gate
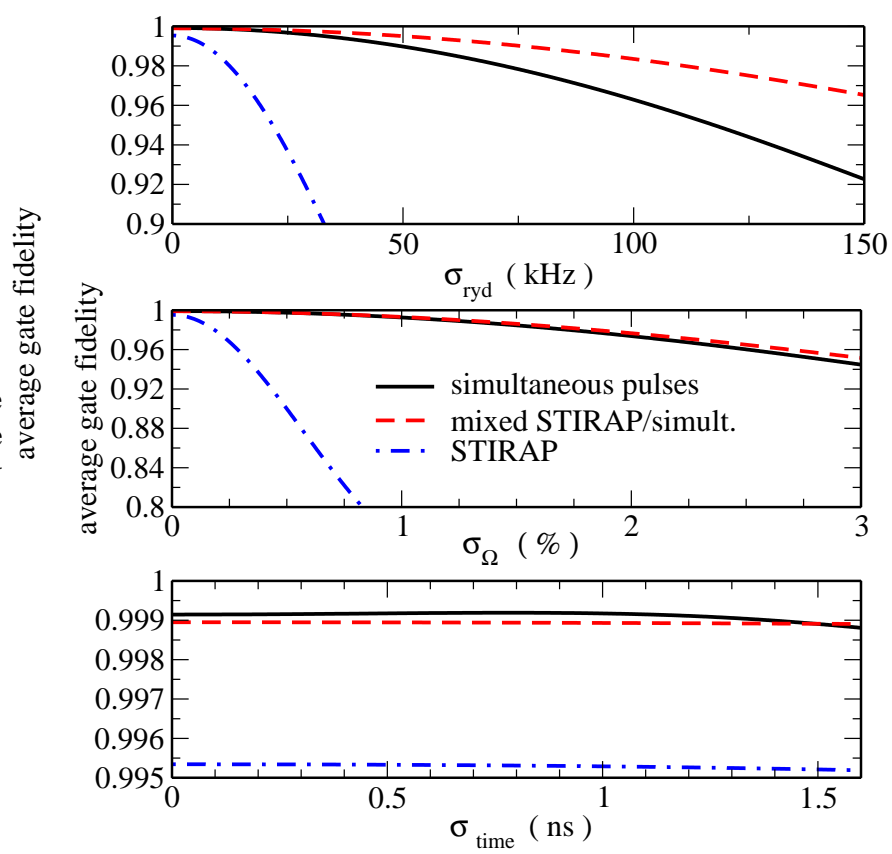

FIG. 9: Robustness of the Rydberg gate with respect to Rydberg level fluctuations (top), amplitude fluctuations (middle), and fluctuations in the relative timing between pulses acting on the left and right atom. All fluctuations are drawn from a Gaussian distribution of width $\sigma_{\text {ryd }}, \sigma_{\Omega}$, and $\sigma_{\text {time }}$, respectively.

fidelity is given by

$$
\tilde{F}\left(\sigma_{x}\right)=\int \frac{1}{\sqrt{2 \pi \sigma_{x}^{2}}} e^{-\frac{\Delta x}{2 \sigma_{x}^{2}}} F(\Delta x) \mathrm{d} x,
$$

with $\sigma_{x}=\sigma_{\text {time }}, \sigma_{\Omega}, \sigma_{\text {ryd }}$, and $\Delta x=\Delta_{\text {time }}, \Delta_{\Omega}, \Delta_{\text {ryd }}$, and $F$ given by Eq (6). Sampling over 1000 variations of each parameter allows to evaluate the integral in Eq. (9) numerically.

Figure 9 shows the resulting expectation value of the gate fidelity vs. standard deviation of the fluctuations in pulse timings, pulse amplitudes, and energy of the Rydberg level. The gate is found to be very robust with respect to pulse timings and fairly robust with respect to amplitudes: only errors of more than a few nanoseconds in timing and several per cent in amplitude reduce the gate fidelity appreciably. A larger sensitivity is found with respect to the position of the Rydberg level: Fluctuations on the order of $1 \%$ of the interaction energy $u$ reduce the gate fidelity to around 0.5 even for the most robust scheme, and even those on the order of $0.1 \%$ of $u$ reduce the fidelity appreciably, cf. top panel of Fig. 9 , This is not surprising, since a 'wrong' energy of the Rydberg level leads to a non-zero two-photon detuning, $\Delta_{2}$, and thus affects both the population transfer for the left atom and the non-local phase accumulated during the pulse acting on the right atom. This additional phase is by assumption unknown and thus cannot be accounted for. Depending on the choice of the Rydberg level, the 
fluctuations of the level energy may be suppressed down to $100 \mathrm{kHz}$ or less [30]. Gate fidelities of about 0.98 are then within reach, cf. the upper panel of Fig. 9.

Though all the schemes behave similarly to variations in timing, there are significant differences in each scheme's robustness to fluctuations in pulse amplitude and Rydberg level energy. For inaccuracies in pulse amplitude, cf. Fig. 9 (middle), the fidelity achieved with STIRAP pulses (dot-dashed blue line) is far more susceptible to small variations than both other schemes. This is due to the additional phase accumulated for STIRAP during the central pulse acting on the right atom, caused by undesired population entering $|r i\rangle$, cf. section IIIB. The mixed scheme (dashed red line) performs slightly better than the simultaneous scheme (solid black line) in this respect, as the robust STIRAP pulses acting on the left atom can achieve efficient population transfer at a wide variety of amplitudes. With respect to fluctuations in the energy of the Rydberg level, in Fig 9 (top) the longer a given scheme populates $|r 0\rangle$, the less robust that scheme is. When the population is in the detuned $|r 0\rangle$ state, it accumulates an undesired phase, and this, not the loss in population transfer efficiency, is the primary reason for the drop in fidelity. The longer a scheme remains in $|r 0\rangle$, the longer it takes to accumulate this additional phase. The mixed scheme, which overlaps the pulses acting on the left and right atom and thus populates $|r 0\rangle$ for the shortest time possible is the most robust to fluctuations in the Rydberg level energy. This is followed by the simultaneous scheme, which fully populates $|r 0\rangle$ for $700 \mathrm{~ns}$, and finally the STIRAP scheme, which fully populates $|r 0\rangle$ for 4200 ns. Counter-intuitively, then, the schemes actually are less robust with respect to variations in Rydberg level energy the longer they become.

\section{OPTIMAL CONTROL}

The use of optimal control theory (OCT) allows to obtain non-analytic pulses that are not bound by conditions of adiabaticity, and can realize gate times at the quantum speed limit [16, 21, 31]. Here, we extend the application of optimal control to increase the robustness of the pulses with respect to fluctuations in amplitude and the energy of the Rydberg level due to external fields. This is achieved by requiring the gate fidelity, Eq. (6), to be close to one not only for the ideal Hamiltonian $\hat{\mathbf{H}}_{0}$, Eq. (2), but also for an ensemble of perturbed Hamiltonians $\left\{\hat{\mathbf{H}}_{i}\right\}, i=[1, N-1]$ that sample the relevant parameter space of variations. Unlike in the analytical pulse schemes, the optimized control pulses will not consist of sub-pulses, but will be completely overlapping. Therefore, an analysis of the robustness with respect to pulse timing is not meaningful in this context. Since population in the intermediate state $|i\rangle$ should be avoided, we perform the optimization in Liouville space, and include spontaneous emission for the intermediate level with a lifetime of $150 \mathrm{~ns}$ [32, 33]. The optimization functional to be minimized reads

$$
J=1-\sum_{n=0}^{N-1} \sum_{i=1}^{16} \mathfrak{R e}\left\{\operatorname{tr}\left[\hat{\mathbf{O}} \hat{\boldsymbol{\rho}}_{i}(0) \hat{\mathbf{O}}^{\dagger} \hat{\boldsymbol{\rho}}_{i, n}(T)\right]\right\}-\sum_{j=1}^{4} \lambda_{j} \int_{0}^{T} \frac{\left(\Omega_{j}(t)-\Omega_{j, \mathrm{ref}}(t)\right)^{2}}{S(t)} \mathrm{d} t
$$

where $\mathbf{O}$ is the CPHASE gate, up to a trivial global phase due to the natural time evolution of the $|1\rangle$ state; the set of $\hat{\boldsymbol{\rho}}_{i}$ matrices are the canonical basis elements of the twoqubit Liouville space, $\{|i\rangle\langle j|\} \forall i, j \in\{00,01,10,11\} ; \lambda_{j}$ are arbitrary positive scaling parameters; the $\Omega_{j}(t)$ are the four controls, i.e. the fields of the red and blue lasers for the left and right atom, respectively; $S(t)$ is a shape function that ensures a smooth switch-on and switch-off of the pulses; and the $\Omega_{j, \text { ref }}$ are a set of reference fields. The gate duration $T$ is fixed for the optimization, but can be systematically varied in order to determine the quantum speed limit. For numerical efficiency, the full basis of 16 states can be replaced by just two density matrices specifically tailored to the optimization problem [34]. The time dependent states $\hat{\boldsymbol{\rho}}_{i, n}(t)$ are determined by the equation of motion,

$$
\frac{\partial}{\partial t} \hat{\boldsymbol{\rho}}_{i, n}(t)=-i\left[\hat{\mathbf{H}}_{n}(t), \hat{\boldsymbol{\rho}}_{i, n}(t)\right]+\mathcal{L}_{D}\left(\hat{\boldsymbol{\rho}}_{i, n}(t)\right)
$$

with $\hat{\boldsymbol{\rho}}_{i, n}(t=0)=\hat{\boldsymbol{\rho}}_{i}(0)$, and $\mathcal{L}_{D}$ the dissipator describing the spontaneous decay from the intermediate level,

$$
\mathcal{L}_{D}(\hat{\boldsymbol{\rho}})=\frac{1}{\tau} \sum_{i=1,2}\left(\hat{\mathbf{A}}_{i} \hat{\boldsymbol{\rho}} \hat{\mathbf{A}}_{i}^{\dagger}-\frac{1}{2}\left\{\hat{\mathbf{A}}_{i}^{\dagger} \hat{\mathbf{A}}_{i}, \hat{\boldsymbol{\rho}}\right\}\right)
$$

with $\hat{\mathbf{A}}_{1}=|0\rangle\left\langle i\left|\otimes \mathbb{1}, \hat{\mathbf{A}}_{2}=\mathbb{1} \otimes\right| 0\right\rangle\langle i|$, and $\tau$ the lifetime.

We use the linear version of Krotov's method [25, 26] to iteratively minimize Eq. (10). If the fields from the previous iteration are used as the reference fields $\Omega_{j, \text { ref }}(t)$, the update equation for each control becomes 


$$
\Delta \Omega_{j}(t)=\frac{S(t)}{\lambda_{j}} \sum_{n=0}^{N-1} \sum_{i=1}^{16} \mathfrak{I m}\left\{\operatorname{tr}\left(-i \hat{\boldsymbol{\sigma}}_{i, n}^{\text {old }}(t)\left[\frac{\partial \hat{\mathbf{H}}_{n}}{\partial \Omega_{j}}, \hat{\boldsymbol{\rho}}_{i, n}^{\text {new }}(t)\right]\right)\right\}
$$
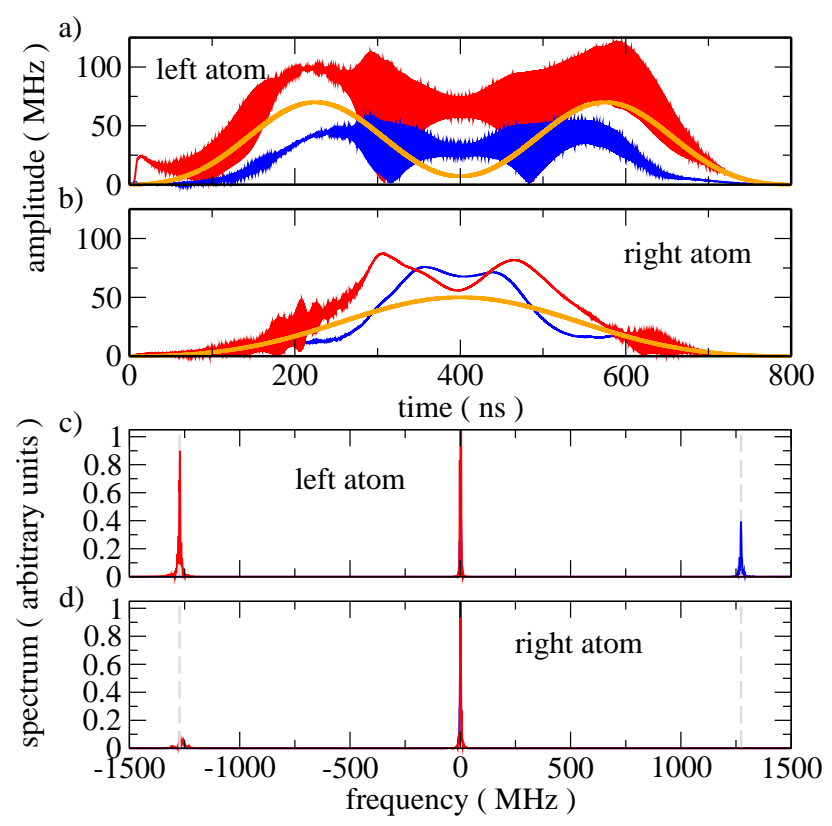

FIG. 10: Amplitudes and spectra of pulses optimized with respect to variations in both two-photon detuning and pulse amplitude, for a gate duration of $T=800 \mathrm{~ns}$. The central peaks in the spectra are truncated to emphasize the sidepeaks. In panel (c), the amplitudes reach a value of 2.0 (red) and 0.8 (blue). In panel (d), the peaks reach 2.5 (red) and 3.0 (blue). Pulses and spectra are shown in the two-color rotating frame. The central frequency of zero corresponds to a laser frequency of the blue pulse that is detuned by $\Delta_{1}$ with respect to the $|0\rangle \rightarrow|i\rangle$ transition. For the red pulse, it indicates the frequency for which there is a two-photon resonance with the $|0\rangle \rightarrow|r\rangle$ transition. The frequencies matching $\pm \Delta_{1}$ are indicated by vertical dashed gray lines.

with the $\hat{\boldsymbol{\sigma}}_{i, n}^{\text {old }}(t)$ being a set of co-states backwards propagated with the pulse from the previous iteration,

$$
\frac{\mathrm{d} \hat{\boldsymbol{\sigma}}_{i, n}(t)}{\mathrm{d} t}=-i\left[\hat{\mathbf{H}}_{n}(t), \hat{\boldsymbol{\sigma}}_{i, n}(t)\right]-\mathcal{L}_{D}\left(\hat{\boldsymbol{\sigma}}_{i, n}(t)\right)
$$

and the 'initial' condition

$$
\hat{\boldsymbol{\sigma}}_{i, n}(t=T)=\hat{\mathbf{O}} \hat{\boldsymbol{\rho}}_{i}(0) \hat{\mathbf{O}}^{\dagger} .
$$

The states $\rho_{i, n}^{\text {new }}(t)$ are forward propagated using the pulse of the current iteration, according to Eq. (11). In the case of the rotating wave approximation where the $\Omega_{j}(t)$ are complex, Eq. (13) is valid for both the real and the imaginary part of the pulse.

In order to optimize for robustness with respect to both amplitude fluctuations and fluctuations of the Rydberg level, we choose an ensemble of $N=24$ Hamiltonians, evenly sampling the values of $\Delta_{r y d}$ between $\pm 300 \mathrm{kHz}$ and variations of the dipole coupling strength between $\pm 5 \%$. The resulting pulses and their spectra are shown in Fig. 10. The guess pulses from which the optimization started are indicated in orange; they are inspired by the analytic scheme of the previous section, consisting of two $\pi$ pulses on the left atom and simultaneously one $2 \pi$ pulse on the right atom. The gate duration was set to $T=800 \mathrm{~ns}$, matching the shortest gate duration obtained for the analytic schemes in the previous section. The choice of the guess pulse is arbitrary in principle, but has significant impact on the convergence speed and the characteristics of the optimized pulse. Indeed, the optimized pulse shapes still roughly follow the shapes of the guess pulses. However, especially for the left atom, there are fast oscillations present in the optimized pulse shapes which correspond to a second laser frequency. As can be seen from the spectra shown in Fig. 10(c), this second frequency is at $+\Delta_{1}$ for the blue pulse and at $-\Delta_{1}$ for the red pulse. In sum, these frequencies still make the pulses two-photon resonant with the $|0\rangle \rightarrow|r\rangle$ transition, providing a second excitation pathway whose interference with the primary pathway can be exploited as a control mechanism. The blue side peak is smaller simply due to the smaller amplitude of that laser. In the spectra of the pulses acting on the right atom, cf. Fig. 10(d), the second frequency is mostly absent, except for the very beginning and end of the red pulse. The population induced by the optimized pulses with the ideal Hamiltonian $\hat{\mathbf{H}}_{0}$ is shown in Fig. 11, Even though the optimized pulses have frequency components that are resonant with the $|0\rangle \rightarrow|i\rangle$ transition, the intermediate level is never significantly populated, due to destructive interference. Suppression of the intermediate state population may be aided by the STIRAP-like feature of the optimized pulse shape, in Fig. 10(a) and (b), where the red laser (counter-intuitively) precedes the blue laser in the initial depopulation of the $|0\rangle$ level of the left atom, and follows it in the final repopulation. Furthermore, the population of the $|01\rangle$ stays remarkable constant, despite the rather large amplitudes of the laser fields in Fig. 10(a). Again, this is due to the interfering multiple pathways. In contrast, the dynamics of the $|10\rangle$ state is much more straightforward, correspondent to the absence of the second laser frequency, and consists effectively of a single $2 \pi$ pulse, although not with full population transfer. The Rydberg blockade is almost fully maintained, cf. the lack of population in the $|r r\rangle$ state in the bottom panel of Fig. 11], Also, the right atom in the time evolution of the $|00\rangle$ state is almost unaffected by the pulse 


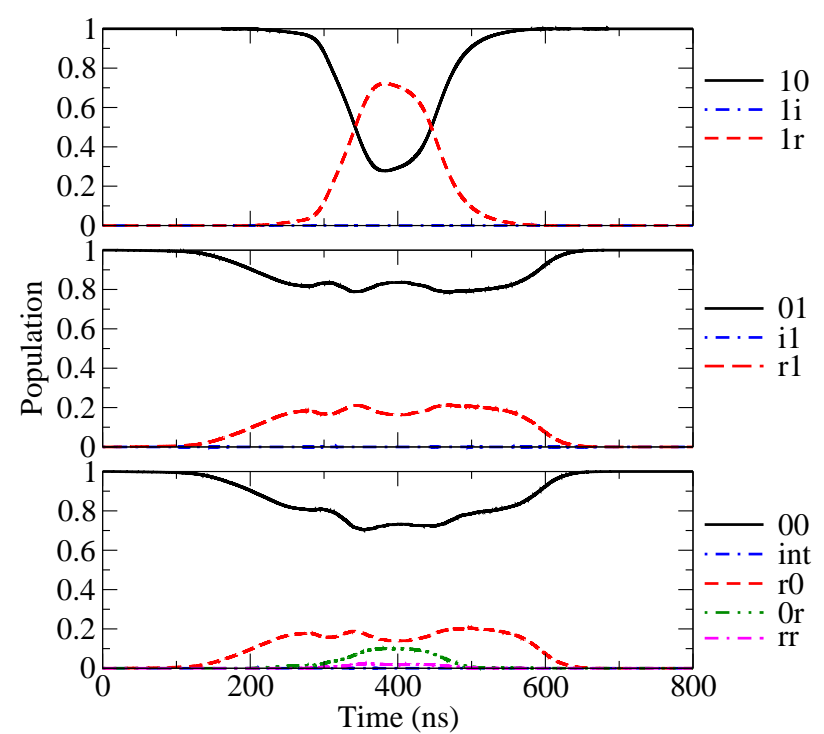

FIG. 11: Dynamics under the pulses optimized with respect to fluctuations in both the Rydberg level and pulse amplitudes, as shown in Fig. 10. The intermediate population in the bottom panel ("int") is integrated over the states $|0 i\rangle$, $|i 0\rangle,|i i\rangle,|i r\rangle$, and $|r i\rangle$. The shown dynamics implement the desired CPHASE gate up to a gate error of $1.04 \cdot 10^{-4}$.

on the right atom, resulting in very similar population dynamics for the $|00\rangle$ and $|01\rangle$ states.

Optimal control also holds the promise of finding pulses approaching the quantum speed limit. We can find solutions with gate durations far below $T=800 \mathrm{~ns}$ required for the analytic schemes, although very short pulses may require unfeasibly large pulse amplitudes. The pulses and spectra resulting from an optimization for $T=100 \mathrm{~ns}$ are shown in Fig. 12. The pulse are optimized for robustness, using the same ensemble of Hamiltonians as for the $T=800 \mathrm{~ns}$ pulses. The pulse shapes again follow the features of the guess pulse, and are only slightly more complex than those for $800 \mathrm{~ns}$ in Fig. 10. The spectra in Fig. 12 (c) and (d) reveal that a similar mechanism as for $T=800 \mathrm{~ns}$ is used to produce the gate, through the presence of additional frequencies at $\pm \Delta_{1}$. The most significant difference to Fig. 10 is that now the additional frequencies are present for both the left and the right atom throughout the entire gate duration. The peaks in the spectrum are broadened due to the shorter time window. Also, the pulse amplitudes are now significantly higher. Generally, the optimization becomes harder for shorter pulse durations, which is why the available control mechanism must now be used more efficiently, thus causing the presence of the second laser frequency throughout all pulses.

The population dynamics, shown in Fig. 13, reflect the increase in the laser amplitudes through some significant differences compared to the dynamics shown in Fig. 11. Most importantly, the Rydberg blockade is now broken, resulting in a significant population of the $|r r\rangle$ state, cf.
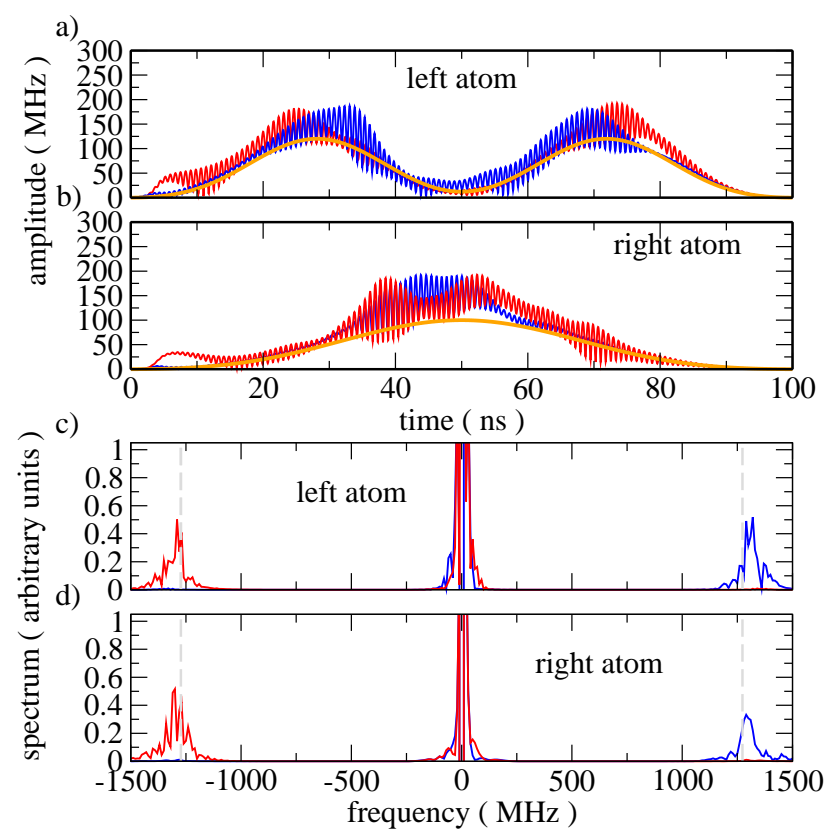

FIG. 12: Amplitudes and spectra of pulses optimized with respect to variations in both two-photon detuning and pulse amplitude, for a gate duration of $T=100 \mathrm{~ns}$. The spectra are drawn on the same scale as in Fig. 10, with the central peaks in panel (c) reaching 4.5 (blue) and 3.0 (red), and 4.5 for both pulses in panel (d).

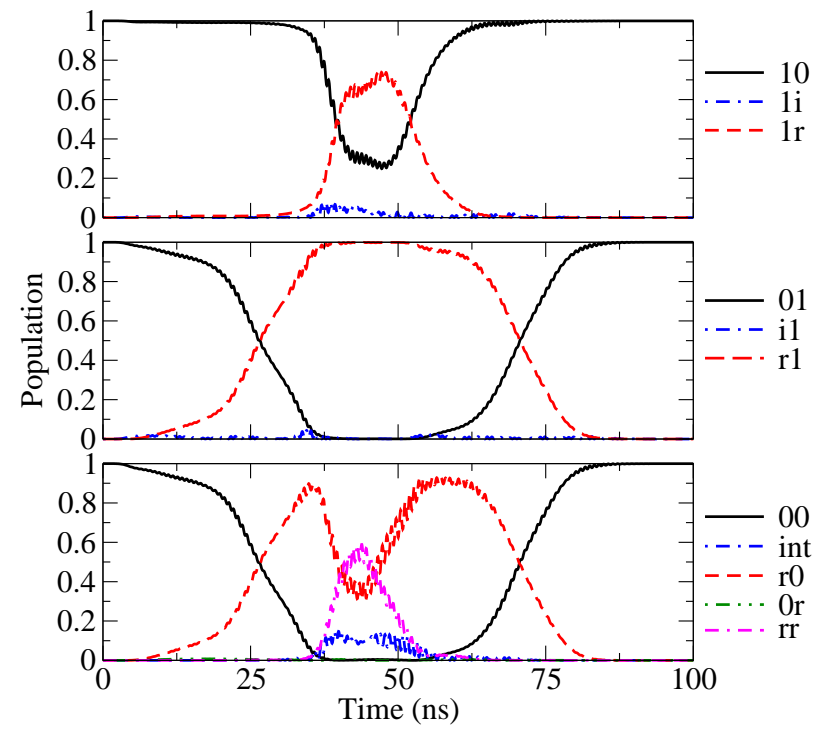

FIG. 13: Dynamics under the optimized pulses shown in Fig. 12. The gate error is $1.92 \cdot 10^{-4}$.

the purple curve in the bottom panel. This nicely illustrates the power of OCT; while the analytic schemes rely on maintaining the blockade regime, the optimization has no such restrictions, and will exploit any pathways available in the time evolution generated by the two-qubit Hamiltonian. There is some minor population 


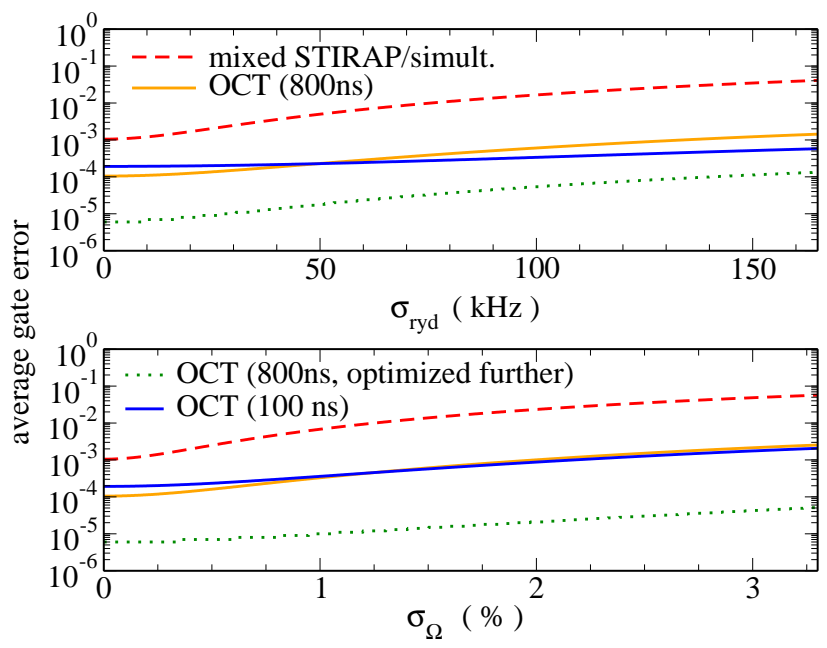

FIG. 14: Expectation value of the gate error in the presence of fluctuations in the $|r r\rangle$ state due to DC electric fields (top), and pulse amplitude fluctuations (bottom). The red dashed curve shows the most robust analytical pulse, cf. the red dashed curve in Fig. 9] The solid yellow and blue lines are for the optimized pulses shown in Figs. 10, 12, respectively. The dotted green line is for a further optimized pulse at $T=800 \mathrm{~ns}$, without any consideration of limits on the pulse amplitude or complexity. Note that both panels show the robustness for same set of pulses, i.e. the pulses were optimized with respect to both variations in the two-photon detuning and the pulse amplitude.

in the intermediate states during the propagation of the $|00\rangle$ state, cf. the blue line in the bottom panel of Fig. 13. However, since the dynamics result from an optimization that took into account the spontaneous decay from the intermediate level explicitly, we are guaranteed that the population in this level is below a threshold that will affect the gate fidelity.

In Fig. 14, we compare the effect of fluctuations on the gate fidelity for the pulses obtained with OCT, cf. Figs. 10and 12 to that for the most robust gates achieved with the analytic schemes, i.e. the mixed scheme employing STIRAP for the pulses on the left atom, and simultaneous pulses for the right atom, cf. Fig. 9. The optimized pulses are significantly more robust with respect to both sources of error by at least an order of magnitude, with the gate fidelity staying above $99.9 \%$ even for large variations, whereas for the analytic pulses, it drops below $97 \%$ for fluctuations of the Rydberg level (top panel) and 95\% for amplitude fluctuations (bottom panel). Note that in contrast to the analytic mixed scheme, the optimized pulses do not require unfeasibly large pulse amplitudes. In contrast, the scheme using only simultaneous pulses but more realistic pulse amplitudes would be even more sensitive - particularly to fluctuations of the Rydberg level (cf. the drop to $92 \%$ gate fidelity in the top panel of Fig. 9). The price for this additional robustness offered by the numerically optimized pulses is a slightly more complex pulse shape and the presence of a second frequency.

It is important to note that the solutions provided by OCT are not unique; the pulses obtained depend on the guess pulses, the exact choice of optimization functional, and on arbitrary scaling parameters such as the $\lambda_{j}$ in Eq. (10). By tuning these parameters carefully, the optimization may be steered towards desired pulse features. It is also possible to add additional constraints to the optimization functional in order to preselect optimization pathways [35]. For example, the $|r r\rangle$ state could be defined as a forbidden subspace in order to enforce the blockade regime, if so desired. One could also include spectral constraints to impose a prespecified pulse bandwidth or suppress undesired frequency components 35, 36]. Optimizing to extremely high fidelities often leads to very large pulse amplitudes or complex pulse features that are undesirable from an experimental point of view. Thus, it is usually best to stop the optimization as soon as the reached fidelities are "good enough", as was done for the optimized pulses shown as solid blue and yellow lines in Fig. 14. In principle, however, pulses of much higher fidelity and robustness than those shown here can be found. This is illustrated by the dotted green line in Fig. 14, which shows the result of a further optimization of the pulse for $T=800$ ns. While these pulses achieve a gate fidelity well above that required for fault tolerant quantum computation [37, 38], the resulting highly optimized pulses have unfeasibly large pulse amplitudes of $1100 \mathrm{MHz}$ and $330 \mathrm{MHz}$ for the blue and red laser, respectively.

\section{SUMMARY AND CONCLUSIONS}

We have studied high-fidelity controlled phasegates based on the Rydberg blockade and investigated their robustness with respect to noise due to stray fields causing fluctuations of the Rydberg level as well as experimental inaccuracies in pulse timings and amplitudes. When single site addressability is available, the gate can be completed by a $2 \pi$-pulse on the right atom, preceded and followed by a $\pi$-pulse on the left atom. For practical reasons, the excitation to the Rydberg level uses a two-photon transition, i.e., each of the three pulses is replaced by a pair of pulses with different frequencies. The pulse pairs can be chosen to occur simultaneously or time-delayed, the latter mimicking a STIRAP sequence. For simultaneous pulse pairs, the Rabi frequency of the red and the blue laser must be identical to achieve population inversion [27]. This is not required for STIRAP. The shortest possible gate duration with analytical pulse shapes is found for a combination of STIRAP pulses acting on the left atom and simultaneous pulses acting on the right atom. The gate duration is limited by the blockade condition which restricts the peak amplitude of the pulses. The STIRAP pulses must furthermore fulfill the adiabaticity condition whereas the peak amplitude of the simultaneous pulses is restricted by the requirement of 
adiabatic elimination of the intermediate level. The gate duration can be significantly shortened by utilizing numerical optimal control to determine the pulse pairs. In this case, neither the blockade condition nor the adiabaticity condition are relevant, and the gate duration is limited by the strength of the interaction between two Rydberg atoms.

For an ideal implementation of the pulse sequences, very high fidelities beyond the quantum error correction threshold can be achieved. This is, however, severely compromised when noise and experimental inaccuracies are taken into account. Gates consisting of STIRAP pairs for all three pulses are found to be the most susceptible to noise with amplitude errors of less than $1 \%$ reducing the fidelity to only 0.8 . This surprising result is explained by the sensitivity of the gate to proper phase alignment: While STIRAP ensures robust population transfer, additional corrections are required to compensate undesired phase evolution [4]. Simultaneous pulses and a combination of STIRAP and simultaneous pulses are somewhat more robust. However, also for these pulse sequences, the fidelities are reduced to below 0.95 for realistic noise levels. Of the three noise sources considered, fluctuations of the Rydberg level due to stray fields are the most severe, whereas timing inaccuracies of the order of 1 ns play almost no role.

In order to identify pulse sequences that are inherently robust to noise, we have employed optimal control theory and calculated pulses which guarantee a high gate fidelity as long as the fluctuations of the Rydberg level and pulse amplitude are confined to a predefined tolerance window. For realistic noise levels we were able to generate pulses that yield gate errors well below $10^{-3}$, with errors below $10^{-5}$ being reached when no limits are placed on pulse amplitudes. Optimized pulse sequences are not only more robust but can also be of much shorter duration. For both short and long gates, the optimized pulses require only one more frequency, corresponding to the one-photon detuning, and their temporal shape is comparatively simple. Taking into account the restrictions of feasible pulse amplitudes, we therefore conclude that optimized pulses achieving fault tolerant gates are experimentally realizable.

Our optimized pulses may also point the way for the construction of improved analytical pulse sequences. The additional frequencies identified by the optimization are utilized to build destructive interference in the intermediate level that is most severely affected by spontaneous decay. It thus allows for resonant transitions, decreasing the pulse amplitudes and lifting the requirements due to adiabaticity and adiabatic elimination.

Lastly, the optimization technique presented here may also be able to address robustness of the gate with respect to undesired excitation in the vibrational degree of freedom, which was not considered in this paper. A vibrational excitation could be modeled as a fluctuation of the $|0\rangle$ or intermediary level, which would allow to optimize over an ensemble of different vibrational states.

\section{Acknowledgments}

We thank Dave Weiss, Ted Corcovilos, Mark Saffman and Reinhold Blümel for fruitful discussions. This research was supported by the DARPA QuEST program, the Deutscher Akademischer Austauschdienst and the National Science Foundation under the Catalzying International Collaborations program (Grant No. OISE1158954). We also thank the Kavli Institute for Theoretical Physics for hospitality and for supporting this research in part by the National Science Foundation Grant No. PHY11-25915.
[1] M. Saffman, T. G. Walker, and K. Mølmer, Rev. Mod. Phys. 82, 2313 (2010).

[2] D. Jaksch, J. I. Cirac, P. Zoller, S. L. Rolston, R. Côté, and M. D. Lukin, Phys. Rev. Lett. 85, 2208 (2000).

[3] M. Lukin, M. Fleischhauer, R. Cote, L. Duan, D. Jaksch, J. Cirac, and P. Zoller, Phys. Rev. Lett. 87, 037901 (2001).

[4] I. I. Beterov, M. Saffman, E. A. Yakshina, V. P. Zhukov, D. B. Tretyakov, V. M. Entin, I. I. Ryabtsev, C. W. Mansell, C. MacCormick, S. Bergamini, et al., Phys. Rev. A 88, 010303 (2013).

[5] E. Urban, T. A. Johnson, T. Henage, L. Isenhower, D. D. Yavuz, T. G. Walker, and M. Saffman, Nature Phys. 5, 110 (2009).

[6] A. Gaëtan, Y. Miroshnychenko, T. Wilk, A. Chotia, M. Viteau, D. Comparat, P. Pillet, A. Browaeys, and P. Grangier, Nature Phys. 5, 115 (2009).

[7] T. Wilk, A. Gaëtan, C. Evellin, J. Wolters, Y. Miroshnychenko, P. Grangier, and A. Browaeys, Phys. Rev. Lett. 104, 010502 (2010).
[8] L. Isenhower, E. Urban, X. L. Zhang, A. T. Gill, T. Henage, T. A. Johnson, T. G. Walker, and M. Saffman, Phys. Rev. Lett. 104, 010503 (2010).

[9] S. Bergamini, B. Darquié, M. Jones, L. Jacubowiez, A. Browaeys, and P. Grangier, J. Opt. Soc. Am. B 21, 1889 (2004).

[10] K. D. Nelson, X. Li, and D. S. Weiss, Nature Physics 3, 556 (2007).

[11] S. Whitlock, R. Gerritsma, T. Fernholz, and R. Spreeuw, New J. Phys. 11, 023021 (2009).

[12] W. S. Bakr, J. I. Gillen, A. Peng, S. Fölling, and M. Greiner, Nature 462, 74 (2009).

[13] J. Kruse, C. Gierl, M. Schlosser, and G. Birkl, Phys. Rev. A 81, 060308 (2010).

[14] C. Weitenberg, M. Endres, J. F. Sherson, M. Cheneau, P. Schauß, T. Fukuhara, I. Bloch, and S. Kuhr, Nature 471, 319 (2011).

[15] X. Zhang, A. Gill, L. Isenhower, T. Walker, and M. Saffman, Phys. Rev. A 85, 042310 (2012).

[16] M. H. Goerz, T. Calarco, and C. P. Koch, J. Phys. B 44, 
154011 (2011).

[17] M. Murphy, S. Montangero, T. Calarco, P. Grangier, and A. Browaeys, arXiv:1111.6083 (2011).

[18] E. Brion, L. H. Pedersen, and K. Mølmer, J. Phys. B 40, S159 (2007).

[19] U. Gaubatz, P. Rudecki, S. Schiemann, and K. Bergmann, J. Chem. Phys. 92, 5363 (1990).

[20] D. Møller, L. B. Madsen, and K. Mølmer, Phys. Rev. Lett. 100, 170504 (2008).

[21] M. M. Müller, H. Haakh, T. Calarco, C. P. Koch, and C. Henkel, Quantum Inf. Process. 10, 771 (2011).

[22] T. Caneva, M. Murphy, T. Calarco, R. Fazio, S. Montangero, V. Giovannetti, and G. E. Santoro, Phys. Rev. Lett. 103, 240501 (2009).

[23] V. Giovannetti, S. Lloyd, and L. Maccone, Phys. Rev. A 67, 052109 (2003).

[24] A. Konnov and V. Krotov, Automation and Remote Control 60, 1427 (1999).

[25] J. P. Palao and R. Kosloff, Phys. Rev. A 68, 062308 (2003).

[26] D. M. Reich, M. Ndong, and C. P. Koch, J. Chem. Phys. 136, 104103 (2012).
[27] B. W. Shore, Manipulating Quantum Structures Using Laser Pulses (Cambridge University Press, 2011).

[28] T. Corcovilos and D. S. Weiss, private communication.

[29] K. Bergmann, H. Theuer, and B. W. Shore, Rev. Mod. Phys. 70, 1003 (1998).

[30] M. Saffman, private communication.

[31] M. M. Müller, D. M. Reich, M. Murphy, H. Yuan, J. Vala, K. B. Whaley, T. Calarco, and C. P. Koch, Phys. Rev. A 84, 042315 (2011).

[32] E. Campani, G. Degan, and G. Gobini, Lettere Al Nuovo Cimento 23, 187 (1978).

[33] M. Ortiz and J. Campos, Journal of Quantitative Spectroscopy and Radiative Transfer 26, 107 (1981).

[34] M. H. Goerz, D. M. Reich, and C. P. Koch, arXiv:1310.2271 (2013).

[35] J. P. Palao, D. M. Reich, and C. P. Koch, Phys. Rev. A 88, 053409 (2013).

[36] D. M. Reich, J. P. Palao, and C. P. Koch, J. Mod. Opt. (2013).

[37] D. Gottesman, arXiv:1310.2984 (2013).

[38] B. W. Reichardt, Algorithmica 55, 517 (2009). 